\title{
Patent ductus arteriosus: to treat or not to treat?
}

\section{William E Benitz}

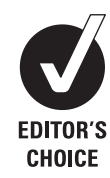

\section{ABSTRACT}

Persistent patency of the ductus arteriosus in the preterm infant is associated with numerous morbidities, including higher rates of bronchopulmonary dysplasia and increased mortality. These strong associations have led to widespread use of cyclooxygenase inhibitors and surgical ligation to achieve ductal closure in the expectation that closing the ductus will reduce these complications. Each of these interventions has its own associated adverse effects. Neither individual randomised controlled trials nor meta-analyses of those trials have been able to demonstrate longterm benefits of these treatments despite their efficacy in inducing ductal closure and reducing the need for ductal ligation. Despite the potential shortcomings of those trials, they provide substantial cumulative evidence that early, routine treatment to close a persistently patent ductus arteriosus in preterm infants does not improve outcomes and should therefore be abandoned. Future trials of these interventions for patent ductus management should address different questions. Persistence of ductal patency should be considered a sign of rather than a direct cause of the several morbidities with which it is clearly associated. Practitioners should tolerate ductal patency and learn to manage its causes and consequences rather than focusing on achievement of ductal closure.

In his seminal 1958 report that a patent ductus arteriosus (PDA) murmur is heard more frequently and for a longer time in preterm infants, Burnard also associated delayed ductal closure with respiratory disease:

In premature babies, . . there was a clear connexion with dyspnoea, and the murmur was not heard unless respiratory distress was present. ${ }^{1}$

Other morbidities, including more severe respiratory distress syndrome (RDS), prolonged assisted ventilation,

Correspondence to Dr William E Benitz, Department of Pediatrics, Division of Neonatal and Developmental Medicine, Stanford University School of Medicine, 750 Welch Road, Suite 315, MC 5731, Palo Alto, CA 94304, USA; benitzwe@stanford.edu pulmonary haemorrhage, bronchopulmonary dysplasia (BPD), necrotising enterocolitis (NEC), renal impairment, intraventricular haemorrhage (IVH), periventricular leukomalacia, cerebral palsy and death, were soon found to be more prevalent in preterm infants with persistent PDA. ${ }^{2}$ Excessive mortality among infants with PDA persists to the present time. ${ }^{3}$ The strength of these associations, coupled with increasing awareness of the disordered haemodynamics of a large left-to-right shunt into the low resistance pulmonary circulation, led to the hypothesis that prolonged ductal patency had a causal role in these morbidities.

Reports of surgical ligation of the ductus in preterm infants in the early 1970s were followed by numerous confirmations that ligation could be accomplished without excessive perioperative mortality. Descriptions of induced ductal closure by non-steroidal anti-inflammatory drugs by Friedman et $a l^{4}$ and Heymann et a $t^{5}$ in 1976 were followed by numerous studies confirming that indomethacin and ibuprofen effect ductal constriction and closure, particularly during the first week after birth. These successes were followed by widespread adoption of aggressive measures to ensure early ductal closure in preterm infants.

Treatments to achieve ductal closure have a number of associated morbidities. In contrast to the anticipated prompt respiratory improvement, surgical ligation is often associated with impaired left ventricular systolic function, sometimes resulting in circulatory and respiratory collapse requiring marked escalation in intensive care support. ${ }^{6}$ In a randomised controlled trial, prophylactic ligation increased the risk of bronchopulmonary dysplasia. ${ }^{7}$ Surgical ligation is also associated with diaphragmatic paresis, ${ }^{8}$ life-long paresis of the left vocal $\operatorname{cord}^{9}$ and late development of scoliosis. ${ }^{10}$ Randomised trials of early indomethacin demonstrated prolongation of ventilator support, ${ }^{11}$ worse oxygenation and increased surfactant requirements, ${ }^{12}$ and requirements for higher mean airway pressures ${ }^{13}$ and inspired oxygen concentrations. ${ }^{13} 14$
Treatment with indomethacin has been associated with spontaneous intestinal perforation, ${ }^{15}$ impaired renal function ${ }^{16}$ and altered cerebrovascular autoregulation. ${ }^{17}$ Similar effects have been seen with ibuprofen, ${ }^{18}$ although adverse effects may be less frequent. Downstream effects of early exposure to cyclooxygenase (COX) inhibitors on definitive ductal closure have not been fully explored. Intervention to close a PDA is not entirely benign.

Excluding trials such as those comparing indomethacin with ibuprofen or short with long courses of indomethacin, in which ductal closure was achieved equally in both treatment groups, 49 randomised controlled trials of PDA closure in preterm infants, including nearly 5000 subjects, have been published. ${ }^{2}$ Although nearly all of these trials were primarily designed to assess effects on ductal patency or IVH, all reported data on one or more secondary outcomes. Neither individual trials nor meta-analyses have demonstrated long-term benefits of measures to close the PDA. Cochrane reviews of prophylactic surgical ligation, ${ }^{19}$ indomethacin $^{20}$ or ibuprofen, ${ }^{21}$ of PDA treatment with indomethacin ${ }^{22}$ or ibuprofen, ${ }^{23}$ or of surgical versus medical PDA closure ${ }^{24}$ all found that benefits were limited to ductal closure, fewer ductal ligations, and - with prophylactic indomethacin - less IVH (IVH > grade II) and periventricular leukomalacia. ${ }^{20}$ These neuroimaging effects were not associated with better neurodevelopmental outcomes. Other meta-analyses were also unable to identify beneficial effects, irrespective of whether the criteria for study inclusion were permissive or rigorous (as in the Cochrane analyses) or how trials were grouped for meta-analysis (by treatment, timing, era before or after surfactant, or other aspects of trial design). ${ }^{2} 25$ CIs for effects on the most important outcomes (death, BPD, death or BPD, NEC, developmental delay, neurosensory impairment, and death or neurosensory impairment) include 1 (no effect) and are narrow (reflecting a low probability that the effect size deviates much from 1). This is not an absence of evidence for a benefit from early, routine ductal closure, but rather substantial evidence for an absence of benefit.

This conclusion has three important implications. First, routine treatment to induce early closure of a persistent PDA in preterm infants should be abandoned, because it does not help these babies. Second, more similar clinical 
trials are not needed, and may be inappropriate, because addition of another trial can move the pooled CIs away from the point estimate of no effect only if it enrols many subjects and demonstrates a substantial effect. If yet another trial must be conducted to convince those who believe that routine treatment with COX inhibitors followed by ligation when COX inhibitors fail is the current standard regimen, it should be designed to demonstrate non-inferiority of avoiding those measures, in the context of standardised approaches to other relevant aspects of care, such as fluid management, respiratory care and transfusion guidelines. Third, the concept that a PDA is, in itself, harmful to preterm infants should be set aside. If that were so, closing the ductus, which was consistently achieved in the reported trials, should reduce harmful effects, but it does not. Delayed ductal closure in preterm infants must be a reflection of some underlying process, such as a systemic inflammatory response, that both delays ductal closure and produces the various morbidities that unquestionably are covariant with PDA.

If closing the ductus is not helpful, what are we to do with these babies? The observation that a particular class of treatments (intervention to close the $\mathrm{PDA}$ ) fails to improve outcomes does not mean that no treatment is useful or necessary, or that the PDA can simply be ignored. Other treatments may improve outcomes without inducing ductal closure. These might fall into two broad categories. First, insight into why the ductus remains open in some preterm infants may lead to interventions to alter the natural history of an underlying condition. If a systemic inflammatory response is responsible for both ductal patency and other complications of prematurity, for example, immunomodulatory measures might prove useful. Second, the haemodynamic consequences of a large left-to-right ductal shunt may require active management. ${ }^{26}$ Excessive pulmonary blood flow might be reduced by distending airway pressure, permissive hypercapnia, minimising inspired oxygen concentrations, or transfusion to maintain haematocrits near or above $50 \%$. These measures may also increase systemic cardiac output, ameliorating potential effects of brain, bowel or renal ischaemia. Other measures, including assurance of adequate preload, use of cardiotonic agents or systemic afterload reduction, may also be useful. Judicious fluid restriction may help prevent systemic and pulmonary oedema, as well as promote ductal closure, but must be balanced against compromised cardiac output. Prevention or correction of hypoproteinaemia by optimising protein intake or administration of plasma, may reduce interstitial fluid fluxes, which may be especially salutary in the lungs. These measures require systematic evaluation in controlled clinical trials.

Several conclusions should not be drawn from the negative meta-analyses. It would be wrong to conclude that there are no very low birthweight infants who might benefit from ductal closure. Unfortunately, we do not know precisely how to identify them or when or how to treat them. Because the available data come from trials of early interventions, typically before age 10-14 days, and many control infants received 'back up' or 'rescue' treatment later in their course, it is quite plausible that infants with a persistent PDA in the third or fourth week after birth may benefit from ductal closure. Those with signs of congestive heart failure, pulmonary congestion or renal ischaemia are obvious candidates, but empiric data to inform treatment criteria are lacking. Retrograde diastolic flow in the descending aorta is associated with an increased risk of NEC in term infants with congenital heart disease. ${ }^{27}$ This haemodynamic disturbance has been demonstrated in preterm infants with PDA, but its relationship to risk of NEC in preterm infants remains hypothetical. Development of echocardiographic, clinical or laboratory criteria for either early or delayed intervention is an important goal, and progress is being made in this area. ${ }^{28}$ If we are to avoid repeating the errors of the past, it will be essential to complete the full sequence of investigations, demonstrating that new criteria predict continued ductal patency, correlate with adverse outcomes and identify a cohort in which those outcomes can be ameliorated or averted by closing the ductus. Until the results of such work are available, however, management of these infants will have to be guided by clinical judgement, informed, as much as possible, by pathophysiology rather than direct evidence. Nonetheless, a more conservative approach in which intervention to close the ductus is deferred until the third or fourth week after birth will intrinsically reduce the proportion of infants who receive treatment, since spontaneous ductal closure will occur in a large proportion of infants (especially those weighing $>1000 \mathrm{~g}$ at birth $^{29}$ ). Finally, data gathered from preterm infants have no bearing on management of term infants with a persistent PDA, particularly in the context of congenital heart disease or other syndromic anomalies. Use of COX inhibitors or ligation in those infants must be guided by experience in infants with similar diagnoses.

There is still a great deal to be learned about the natural history of ductal closure in preterm infants, but it is time to reassess our long-held conviction that a patent ductus is a source of rather than a sign of trouble for these infants. As we learn to live with patency of the ductus, we can hope to learn how best to manage both its causes and consequences. Much hard work lies ahead. Until that work is done, we are well advised to follow the example of Clyman and colleagues $^{30}$ in moving incrementally towards less aggressive, more conservative approaches to management of the PDA in preterm infants.

Contributors WEB was solely responsible for conception and design, analysis and interpretation of data, drafting and revising the article, and final approval of the version to be published. No others participated in any phase of creation of this work.

Competing interests None.

Provenance and peer review Commissioned; externally peer reviewed.

Accepted 24 August 2011

Published Online First 15 December 2011

Arch Dis Child Fetal Neonatal Ed 2012;97:F80-F82. doi:10.1136/archdischild-2011-300381

\section{REFERENCES}

1. Burnard ED. A murmur that may arise from the ductus arteriosus in the human baby. Proc $R$ Soc Med 1959;52:77-8

2. Benitz WE. Treatment of persistent patent ductus arteriosus in preterm infants: time to accept the null hypothesis? J Perinatol 2010;30:241-52.

3. Noori S, McCoy M, Friedlich P, et al. Failure of ductus arteriosus closure is associated with increased mortality in preterm infants. Pediatrics 2009;123:e138-44.

4. Friedman WF, Hirschklau MJ, Printz MP, et al. Pharmacologic closure of patent ductus arteriosus in the premature infant. N Engl J Med 1976;295:526-9.

5. Heymann MA, Rudolph AM, Silverman NH. Closure of the ductus arteriosus in premature infants by inhibition of prostaglandin synthesis. N Engl J Med 1976;295:530-3.

6. Moin F, Kennedy KA, Moya FR. Risk factors predicting vasopressor use after patent ductus arteriosus ligation. Am J Perinatol 2003;20:313-20.

7. Clyman R, Cassady G, Kirklin JK, et al. The role of patent ductus arteriosus ligation in bronchopulmonary dysplasia: reexamining a randomized controlled trial. J Pediatr 2009;154:873-6.

8. Yon TF, Amka P, Pildes RS, et al. Diaphragmatic paralysis after surgical ligation of patent ductus arteriosus. Lancet 1977;2:461. 
9. Røksund OD, Clemm H, Heimdal JH, et al. Left vocal cord paralysis after extreme preterm birth, a new clinical scenario in adults. Pediatrics 2010;126:e1569-77.

10. Seghaye MC, Grabitz R, Alzen G, et al. Thoracic sequelae after surgical closure of the patent ductus arteriosus in premature infants. Acta Paediatr 1997;86:213-16.

11. Vincer M, Allen A, Evans J, et al. Early intravenous indomethacin prolongs respiratory support in very low birth weight infants. Acta Paediatr Scand 1987;76:894-7.

12. Yaseen $\mathbf{H}$, al Umran $\mathrm{K}$, Ali $\mathrm{H}$, et al. Effects of early indomethacin administration on oxygenation and surfactant requirement in low birth weight infants. J Trop Pediatr 1997; 43:42-6.

13. Van Overmeire B, Van de Broek H, Van Laer P, et al. Early versus late indomethacin treatment for patent ductus arteriosus in premature infants with respiratory distress syndrome. J Pediatr 2001;138:205-11.

14. Schmidt B, Roberts RS, Fanaroff A, et al. Indomethacin prophylaxis, patent ductus arteriosus, and the risk of bronchopulmonary dysplasia: further analyses from the Trial of Indomethacin Prophylaxis in Preterms (TIPP). J Pediatr 2006;148:730-4.

15. Attridge JT, Clark R, Walker MW, et al. New insights into spontaneous intestinal perforation using a national data set: (1) SIP is associated with early indomethacin exposure. J Perinatol 2006;26:93-9.
16. Vanpée M, Ergander U, Herin P, et al. Renal function in sick, very low-birth-weight infants. Acta Paediatr 1993;82:714-18.

17. Keating $\mathbf{P}$, Verhagen $\mathrm{E}$, van Hoften J, et al. Effect of indomethacin infused over 30 minutes on cerebral fractional tissue oxygen extraction in preterm newborns with a patent ductus arteriosus. Neonatology 2010;98:232-7.

18. Vieux R, Desandes R, Boubred F, et al. Ibuprofen in very preterm infants impairs renal function for the first month of life. Pediatr Nephrol 2010;25:267-74.

19. Mosalli R, Alfaleh K. Prophylactic surgical ligation of patent ductus arteriosus for prevention of mortality and morbidity in extremely low birth weight infants. Cochrane Database Syst Rev 2008;1:CD006181.

20. Fowlie PW, Davis PG. Prophylactic intravenous indomethacin for preventing mortality and morbidity in preterm infants. Cochrane Database Syst Rev 2002;3:CD000174.

21. Shah SS, Ohlsson A. Ibuprofen for the prevention of patent ductus arteriosus in preterm and/or low birth weight infants. Cochrane Database Syst Rev 2006:1:CD004213

22. Cooke L, Steer P, Woodgate P. Indomethacin for asymptomatic patent ductus arteriosus in preterm infants. Cochrane Database Syst Rev 2003;2:CD003745

23. Ohlsson A, Walia R, Shah SS. Ibuprofen for the treatment of patent ductus arteriosus in preterm and/or low birth weight infants. Cochrane Database Syst Rev 2010;4:CD003481.
24. Malviya M, Ohlsson A, Shah S. Surgical versus medical treatment with cyclooxygenase inhibitors for symptomatic patent ductus arteriosus in preterm infants. Cochrane Database Syst Rev 2008:1:CD003951.

25. Jones LJ, Craven PD, Attia J, et al. Network meta-analysis of indomethacin versus ibuprofen versus placebo for PDA in preterm infants. Arch Dis Child Fetal Neonatal Ed 2011;96:F45-52.

26. Benitz WE. Learning to live with patency of the ductus arteriosus in preterm infants. J Perinatol 2011;31(Suppl 1):S42-8.

27. Carlo WF, Kimball TR, Michelfelder EC, et al. Persistent diastolic flow reversal in abdominal aortic Doppler-flow profiles is associated with an increased risk of necrotizing enterocolitis in term infants with congenital heart disease. Pediatrics 2007;119:330-5

28. McNamara PJ, Sehgal A. Towards rational management of the patent ductus arteriosus: the need for disease staging. Arch Dis Child Fetal Neonatal Ed 2007;92:F424-7.

29. Nemerofsky SL, Parravicini E, Bateman D, et al. The ductus arteriosus rarely requires treatment in infants $>1000$ grams. Am J Perinatol 2008:25:661-6.

30. Jhaveri N, Moon-Grady A, Clyman RI. Early surgical ligation versus a conservative approach for management of patent ductus arteriosus that fails to close after indomethacin treatment. J Pediatr 2010;157:381-7, 387.e1. 